\title{
Transverse beam stacking injection system for synchrotron light source booster synchrotrons
}

\author{
R. P. Fliller III, * T. Shaftan, R. Heese, S. Kowalski, J. Rose, and G. Wang \\ Photon Sciences Directorate, Brookhaven National Laboratory, Upton, New York 11973-5000, USA
}

(Received 23 July 2010; published 10 February 2011)

\begin{abstract}
Beam intensity is a key performance parameter for particle accelerators. Modern high-performance storage rings demand injectors that can deliver large currents per injection cycle, which translates to an increase in machine size and consumed power. Therefore, it is justifiable to look for ways to increase the injector's performance while minimizing the size increase. We have investigated a number of ways to accumulate charge in a booster synchrotron and applied one of them to the NSLS-II booster. We have designed a scheme to transversely stack two bunch trains from the injector linac in the booster. In this paper we discuss this stacking scheme. The relevant booster dynamics are studied. The charge transport efficiency of the stacking scheme is studied in detail at injection and through a full booster ramp. Pulsed magnet requirements for the injection system and methods to achieve them are discussed. We show that the charge transport efficiency of the stacking scheme is similar to a single bunch train in the booster. This has become a critical design feature of the NSLS-II booster.
\end{abstract}

DOI: 10.1103/PhysRevSTAB.14.020101

\section{INTRODUCTION}

Beam intensity is a key performance parameter for particle accelerators. Modern high-performance storage rings demand injectors that can deliver large currents per shot, which translates to an increase in machine size and consumed power. Therefore, it is justifiable to look for ways to increase the injector's performance while minimizing the size increase. Beam stacking in a circular machine is a well-known method of accumulating charged particles when their source has limited intensity. This is a routine strategy for storing electrons in synchrotron-light sources. More sophisticated stacking methods include transverse phase-space painting $[1,2]$, longitudinal stacking $[3,4]$, and rf gymnastics [5].

In this paper, we discuss methods of increasing the NSLS-II injector performance by accumulating the bunch trains during the booster ramp. The NSLS-II injection system will feature a $200 \mathrm{MeV}$ linac with thermionic cathode and a $3 \mathrm{GeV}$ booster synchrotron [6-10]. In order to meet the needs of top off injection, the linac needs to provide $15 \mathrm{nC}$ to the booster. The required charge and bunch pattern have yet to be demonstrated from a linac at an operating light source. Existing facilities are capable of producing approximately half of the required charge [11]. We have developed a method to transversely stack two bunch trains in the booster to ensure that the charge requirement can be met. The linac charge output requirement is approximately halved using beam stacking in the booster, and linac operation is improved since the linac does not need to operate at peak current output at all times.

Electron storage rings typically practice injection with stiff, multi-GeV beams. The beam dynamics is dominated

\footnotetext{
*rfliller@bnl.gov
}

PACS numbers: 29.27.Ac, 29.27.Eg, 41.85.Ar, 41.85.Ew

by radiation damping with time scales of a few milliseconds. Therefore injection transients quickly decay and do not contribute to the beam emittance. In the case of the NSLS-II booster, the beam injection energy is $200 \mathrm{MeV}$, the damping time is 15 seconds, and the injection repetition rate is $10 \mathrm{~Hz}$. Therefore, the effect of the injection transients do not decay away. The first bunch train must circulate until the second train arrives from the linac. This is a mode of operation that is not desired for most booster synchrotrons as the beam emittance generally increases. Therefore we have studied the beam dynamics at injection energy as well as extraction energy to ensure that beam stacking is a viable option. We show in this paper that the booster will be able to limit the first injected beam emittance growth to acceptable levels for beam stacking.

The NSLS-II injection scheme has as an essential element the requirement to maintain the accumulated charge. If storage ring injection is lossy, there is always the option of injecting more charge per injection or injecting more frequently. In the case of stacking in the booster, if the charge is not maintained then the entire enterprise fails. Therefore great care must be taken to optimize the various parameters of the booster, the booster injection system, and the beam from the linac. Good transmission of the stacked beam during the booster ramp is critical. We show that the stacked beam emittance uses up to $61 \%$ of the NSLS-II booster acceptance. Orbit errors, beam motion, power supply variations, and the nonlinear fields in the combined function magnets during the early portions of the ramp can easily eat away at the remaining acceptance or cause emittance growth. This can cause enough charge loss such as to make the stacking scheme worse than ramping a single beam. We show in this paper that not only can we place two beams into the booster at injection, but that we 


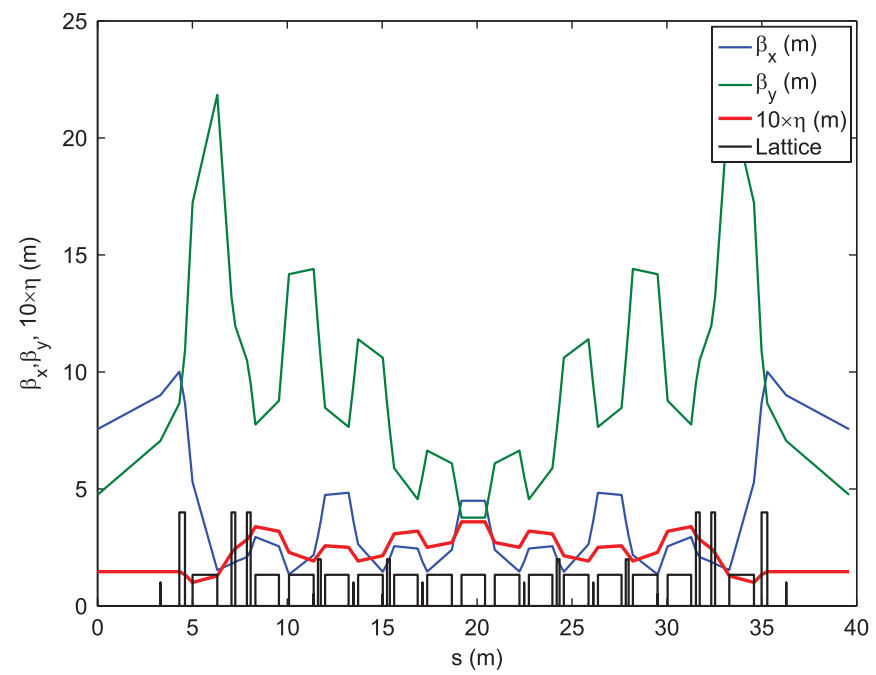

FIG. 1. $\beta$ and dispersion functions for one-quarter of the NSLS-II booster.

can ramp this beam with over $90 \%$ charge transmission in the worst cases.

Modern storage ring magnets are optimized for a very narrow energy range, typically a few percent. Boosters at most light sources need to operate over a range of 15 to 30 in energy. This means that at injection the fields are proportionally lower. The relative field errors are amplified by this same factor of 15 to 30 and can be difficult to measure at low field. The effect of the field errors at low field for beam with betatron amplitudes several times their emittance makes maintaining the beam emittance and charge more difficult than in a storage ring.

In this paper we report on the design of a beam stacking system for the NSLS-II booster. First, we evaluate the stay clear aperture and the dynamic aperture of the booster. Then the geometry of the injection straight and kicker requirements are specified. A booster ramp is simulated to understand the charge transport efficiency of a single bunch train. Tracking is done at the injection porch to understand the behavior of the first bunch train while waiting for the next bunch. Finally, tracking of the stacked train is shown for a variety of initial conditions and the charge transport efficiency is compared to the single bunch train case.

\section{NSLS-II BOOSTER REFERENCE DESIGN}

The NSLS-II injection system will feature a $200 \mathrm{MeV}$ linac and a $3 \mathrm{GeV}$ booster synchrotron. The linac will operate in two modes, long pulse mode and short pulse mode. In long pulse mode the linac is specified to deliver $15 \mathrm{nC}$ of charge per bunch train to the booster with 80-150 bunches separated by $2 \mathrm{~ns}$. Short pulse mode will feature a single to a few bunches separated by arbitrary multiples of $2 \mathrm{~ns}$, with each bunch containing $0.5 \mathrm{nC}$. In each case the transverse geometric emittance is $4 \sigma_{x} \sigma_{x^{\prime}}=150 \mathrm{~nm}$ with $0.5 \%$ energy spread. The linac will have a $10 \mathrm{~Hz}$ repetition rate [12].

The NSLS-II booster is a $158.4 \mathrm{~m}$ circumference synchrotron utilizing combined function magnets. The booster
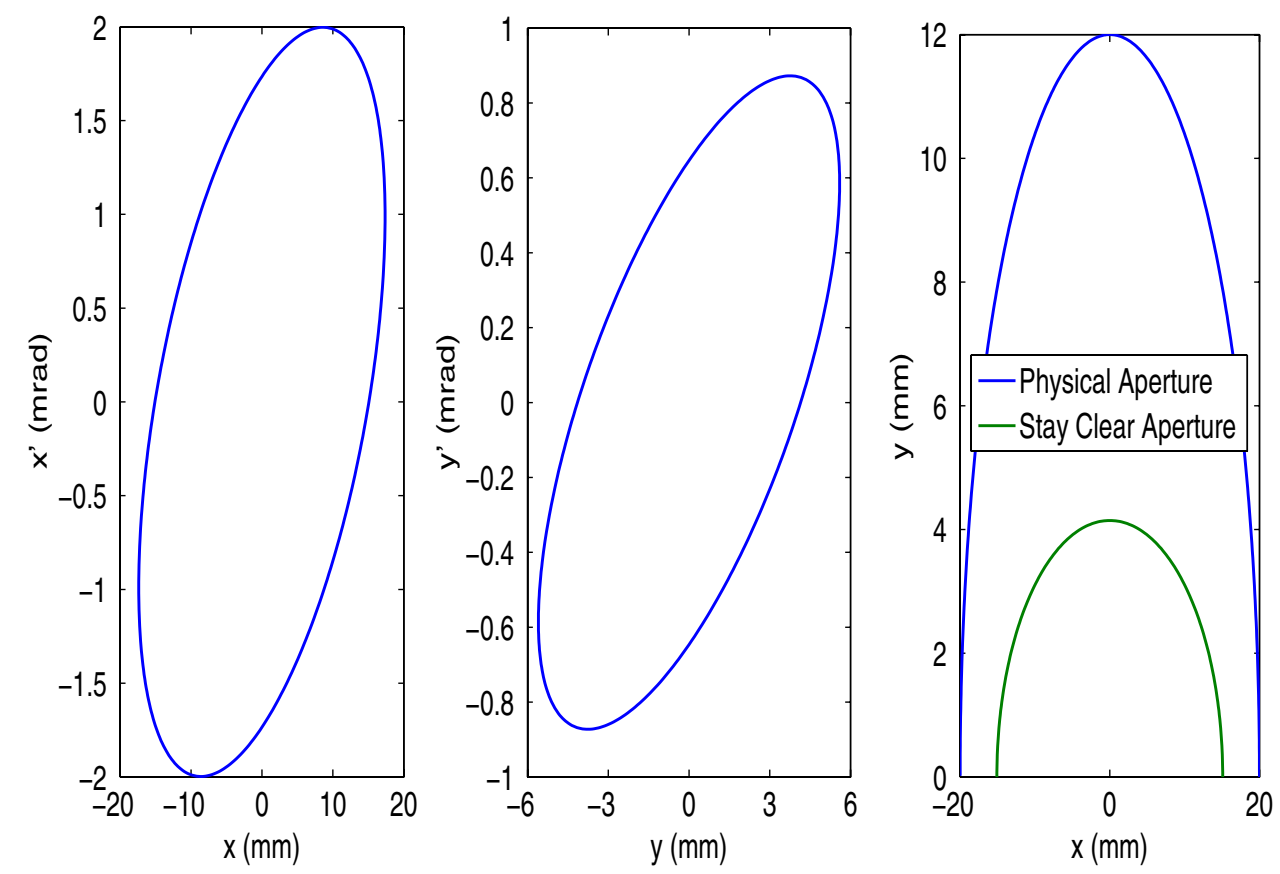

FIG. 2. The stay clear aperture at the end of the injection straight. The left plot is the horizontal phase-space boundary, the center plot is the vertical phase-space boundary. The right plot shows the physical aperture compared to the stay clear aperture at the end of the injection straight. 
is designed to have a $39 \mathrm{~nm}$ equilibrium emittance at $3 \mathrm{GeV}$ to meet the injection requirements for the storage ring. It is divided into four quadrants consisting of 11 FODO cells each. There are four insertions used for injection, diagnostics, extraction, and rf. The lattice functions for one quadrant are shown in Fig. 1 [9].

The booster if system will be a five-cell, $500 \mathrm{MHz}$ PETRA cavity, with space available for an additional cavity in the future [9]. The cavity will be capable of producing 1.2 MV for a bucket height of $0.6 \%$ at $3 \mathrm{GeV}$. The rf voltage is reduced to $150 \mathrm{kV}$ at injection. This corresponds to a bucket height of $1.5 \%$. The linac beam will have an energy spread of $0.5 \%$ FWHM determined by the energy slit in the transport line.

The booster aperture is an ellipse with major and minor axes of $20 \times 12 \mathrm{~mm}^{2}$ in the dipoles. This is chosen to keep the dipole gap small. These apertures place a limit on the machine acceptance. Using the Twiss functions in Fig. 1, one can calculate the available phase space for injection at the end of the injection straight. We call this the stay clear aperture. This is shown in Fig. 2. The stay clear aperture area is $31 \mathrm{~mm}$ mrad in the horizontal, and $3.6 \mathrm{~mm}$ mrad in the vertical $[9,10,13]$. This is much larger than the beam emittance $4 \sigma_{x} \sigma_{x^{\prime}}=150 \mathrm{~nm}$. The acceptance limitations come from the large $\beta$ functions in the quadrupoles at the end of the straight sections. Figure 3 shows the stay clear aperture through one quadrant of the booster. The booster acceptance provides the ultimate limit on what stacking schemes will work and how many bunch trains can be stacked.
TABLE I. Errors used in simulating the booster.

\begin{tabular}{lc}
\hline \hline Parameter & Error \\
\hline Dipole transverse alignment & $100 \mu \mathrm{m}$ \\
Dipole longitudinal alignment & $500 \mu \mathrm{m}$ \\
Dipole roll & $200 \mu \mathrm{rad}$ \\
Dipole field & $0.02 \%$ \\
Dipole gradient & $0.2 \%$ \\
Dipole sextupole component & $2 \%$ \\
Quadrupole transverse alignment & $150 \mu \mathrm{m}$ \\
Quadrupole longitudinal alignment & $500 \mu \mathrm{m}$ \\
Quadrupole gradient & $0.5 \%$ \\
Quadrupole roll & $200 \mu \mathrm{rad}$ \\
Sextupole transverse alignment & $150 \mu \mathrm{m}$ \\
Sextupole longitudinal alignment & $500 \mu \mathrm{m}$ \\
Sextupole roll & $200 \mu \mathrm{rad}$ \\
Beam position monitor transverse alignment & $150 \mu \mathrm{m}$ \\
\hline \hline
\end{tabular}

The dynamic aperture of the booster was also evaluated by $6 \mathrm{D}$ tracking including synchrotron oscillations, radiation damping, and errors [9]. The rms errors used are shown in Table I. Twenty seeds were used. Figure 4 shows the dynamic aperture of the booster at the end of the injection straight at $200 \mathrm{MeV}$ for a single seed. The average dynamic aperture is $1720 \pm 530 \mathrm{~mm}^{2}$ which is larger than the physical aperture and the stay clear aperture so will not be a limiting factor for beam stacking.

The booster dispersion sets a momentum aperture of $5 \%$. As mentioned above, the rf voltage at injection limits the

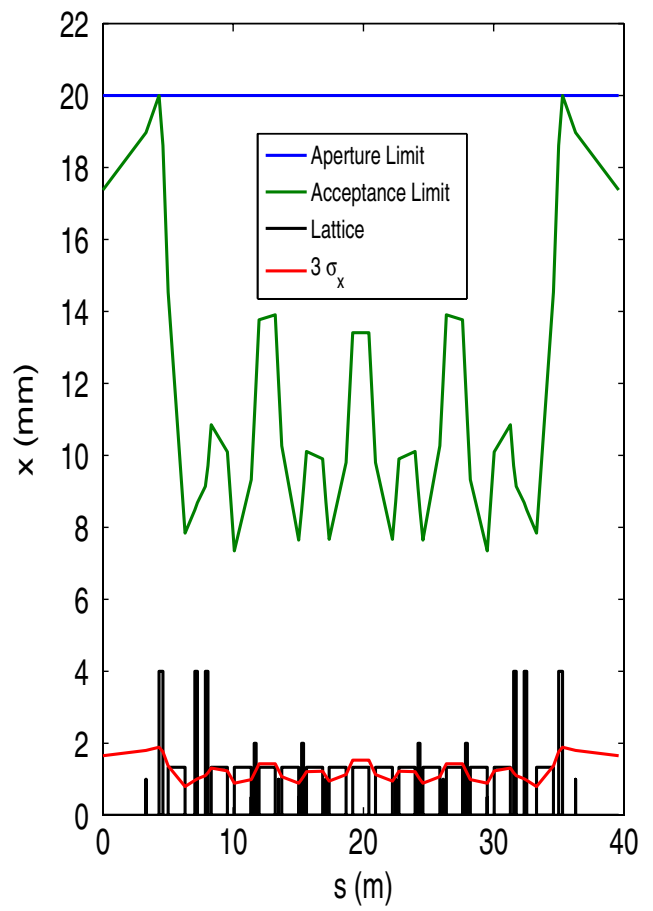

FIG. 3. The horizontal (left) and vertical (right) aperture limit in the NSLS-II booster. 


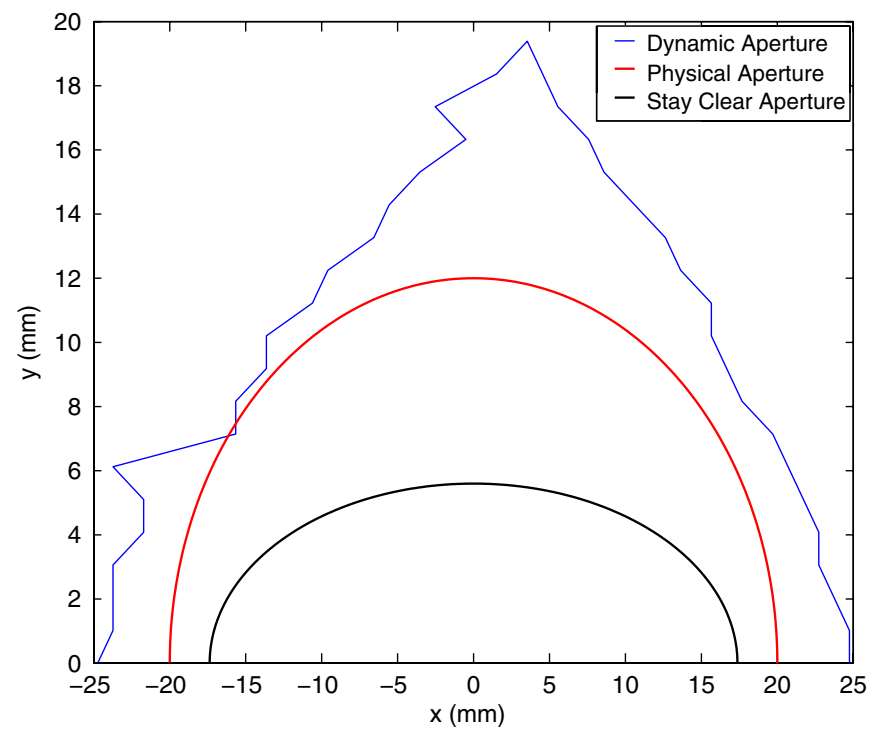

FIG. 4. The dynamic aperture in the booster compared to the physical and stay clear apertures at $200 \mathrm{MeV}$.

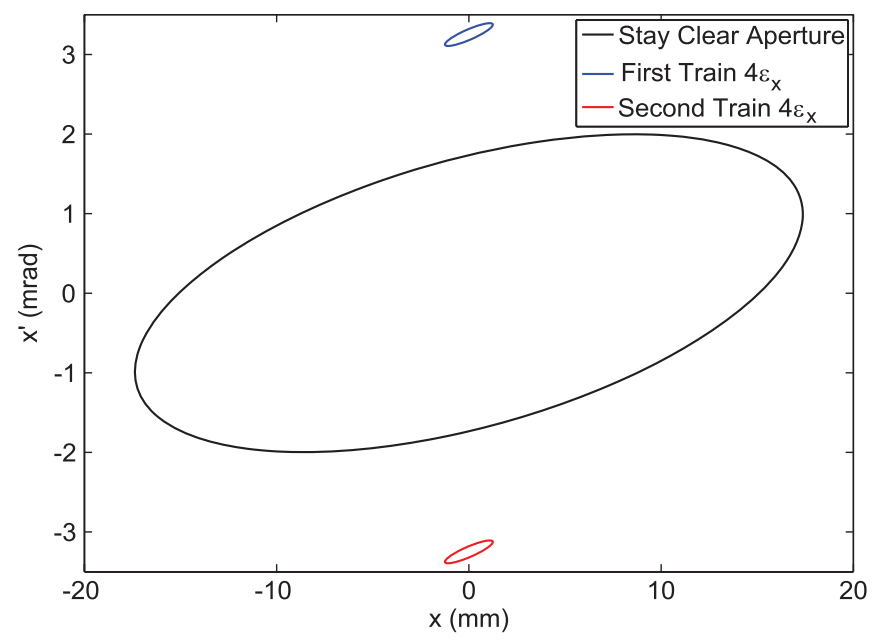

FIG. 5. The horizontal stay clear aperture shown with the stacked train phase ellipses after injection of the second bunch for the initial booster stacking scheme. Both trains lie outside of the stay clear aperture. bucket height to $1.5 \%$, limiting the booster energy acceptance.

\section{BOOSTER INJECTION SYSTEM}

The booster injection straight passed through two design iterations. The initial design of the booster injection straight consisted of a septum and a single kicker magnet separated by $3.8 \mathrm{~m}$. The initial idea to stack the beam with this system proceeds as follows. The first bunch train is injected into the booster. The beam would circulate for $100 \mathrm{~ms}$ until the second bunch arrived. The second bunch would exit the septum and arrive at the kicker in the same way as the first bunch. The kicker would fire at half strength. At this point the beams would be at the same transverse location, but separated by $7.5 \mathrm{mrad}$ in angle. As Fig. 5 illustrates, there is a maximum of $\pm 2 \mathrm{mrad}$ available for injection and the beams lie outside of the stay clear aperture. This stacking scheme was abandoned and the injection straight and transport line were redesigned.

The chosen booster injection straight section design is shown in Fig. 6. The straight contains four fast kicker magnets, and a pulsed septum magnet between the pairs. The kickers are $20 \mathrm{~cm}$ long, capable of kicking the beam $15 \mathrm{mrad}$, and are separated by $2.25 \mathrm{~m}$ to translate the beam $28 \mathrm{~mm}$ horizontally. The injection septum is $75 \mathrm{~cm}$ long, and deflects the beam $125 \mathrm{mrad}$. The knife edge is located $20 \mathrm{~mm}$ from the central orbit.

The injection sequence for the first linac bunch train is as follows. When the beam arrives from the linac, the septum deflects the beam so that it will be $27 \mathrm{~mm}$ from the central orbit with no angle. Bumps BR-BU3SI and BR-BU4SI kick the beam $12 \mathrm{mrad}$ each to place the beam on the central orbit with no angle. This is shown in Fig. 6. If only one bunch train is desired, the booster initiates the ramp.

If a second train is desired, the initial injected beam will circulate on the injection porch for $100 \mathrm{~ms}$ until the next beam from the linac arrives. Bumps BR-BU1SI and BRBU2SI will kick the circulating beam with an angle of $7.1 \mathrm{mrad}$ to move it over $17 \mathrm{~mm}$ toward the septum. The septum fires normally, placing the second train parallel to the first. The beam separation is $10.5 \mathrm{~mm}$ center to center. Bumps BR-BU3SI and BR-BU4SI fire with an angle of

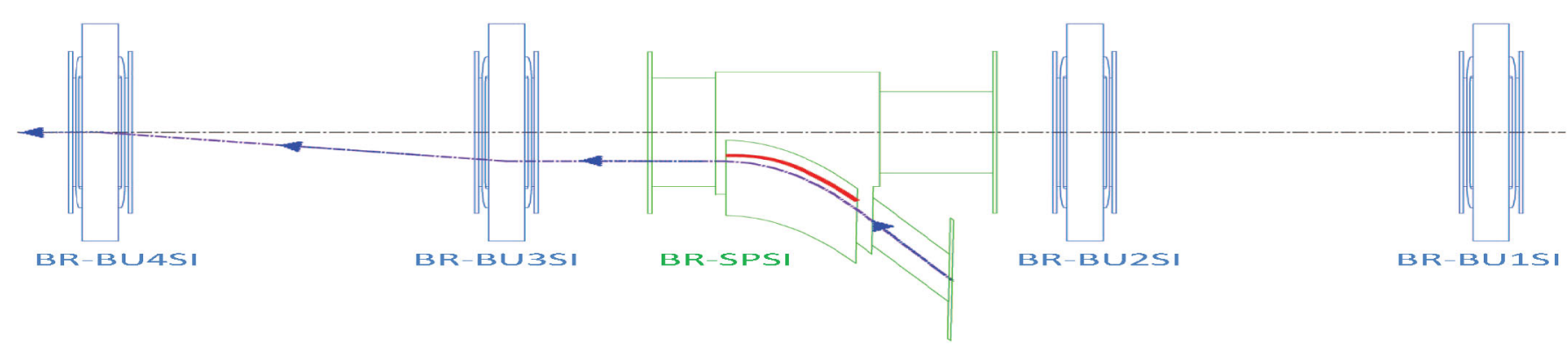

FIG. 6. Schematic of the booster injection straight showing the injection of the first bunch train. The blue magnets are the injection kickers, the green magnet is the septum, the red line denotes the septum knife. 


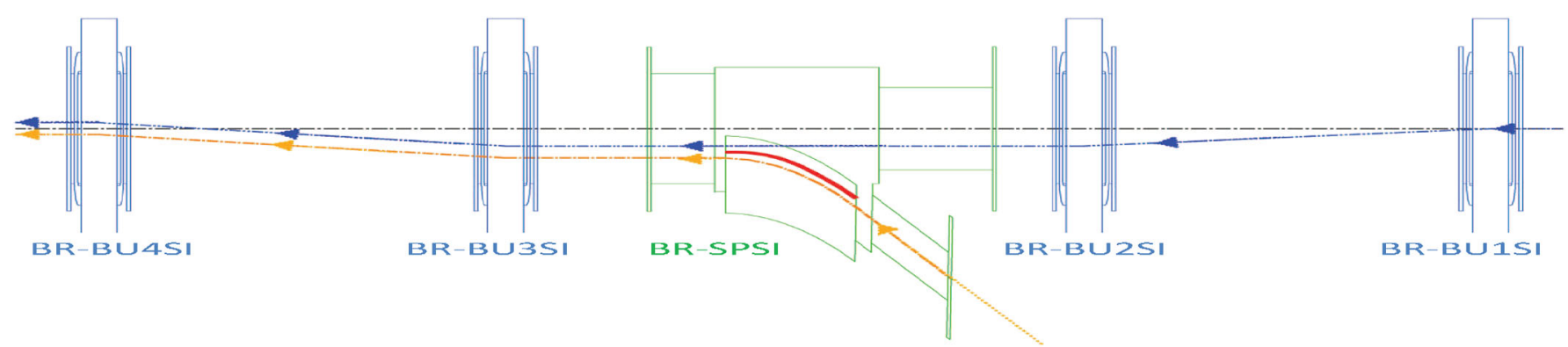

FIG. 7. Schematic of the booster injection straight showing the injection of the second bunch train. The blue magnets are the injection kickers, the green magnet is the septum.

$9.56 \mathrm{mrad}$ each to place the centroid of the two beams on the central orbit with no angle. At the end of the injection straight the two trains are offset from the central orbit by $\pm 5.25 \mathrm{~mm}$. This is shown in Fig. 7. The beams are inside the stay clear aperture as shown in Fig. 8. The booster ramp will start, and the bunches will coalesce through filamentation and radiation damping.

Table II lists the septum magnet, kicker magnet, and driver parameters [14]. Each magnet will be powered by a separate voltage regulated DC power supply. The booster injection kickers will utilize a thyratron switching element as the drive for the system in a low inductance configuration. The thyratron will be triggered from the timing system. A pulse forming line was chosen to generate the flattop current pulse. This will be accomplished using transmission lines stacked in parallel to achieve the desired impedance and to obtain better flattop ripple and droop. There is an impedance matching resistor to the pulse forming line and series diode set to block the reverse voltages of the transmission line. The capacitor chosen for the system will be a trade-off between rise time and possible overshoot. The capacitor can be implemented in

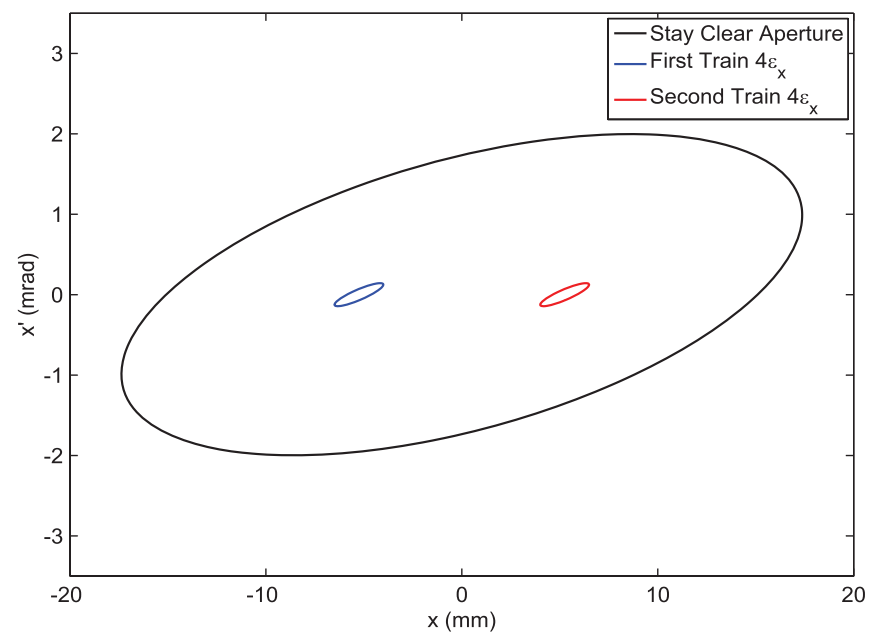

FIG. 8. The horizontal stay clear aperture shown with the stacked train phase ellipses after injection of the second bunch for the final booster stacking scheme. Both trains lie inside of the stay clear aperture. such a way as to give steeper rise times at the magnet while having little overshoot by simply increasing or decreasing the capacitor values. The power supply will be protected by another series set of diodes and a current limiting resistor. One major benefit of the booster injection kicker system is that the thyratron is cathode grounded. The other benefit is the circuit layout can be configured in such a way that allows for easy installation and servicing periods. Figure 9 shows the approach that will be used. Figure 10 shows the simulated pulse for the booster injection kicker.

The present booster injection septum design utilizes the standard configuration which is to discharge a capacitor bank through the magnet which is controlled by a distributed gate thyristor. The positive half sine is created by a silicon controlled rectifier (SCR) and the negative half sine by the freewheeling diode connected across the thyristor. The thyristor and antiparallel diode will be mounted on hardware that is both easy to maintain and is torque adjustable. Across the diode and thyristor there will be a snubber network to dampen any transient spikes. The returned energy will go back to the capacitor bank. A loop will be needed to ensure constant energy at the septum. The basic loop will detect the peak current and compare the set value to the value across the capacitor bank which will be adjusted to account for fluctuations in voltage. This is shown schematically in Fig. 11.

TABLE II. Booster injection pulsed magnet parameters.

\begin{tabular}{lcc}
\hline \hline Parameter & Septum & Kicker \\
\hline Bend angle $(\mathrm{mrad})$ & 125 & 15 \\
Length $(\mathrm{cm})$ & 75 & 20 \\
Aperture $\left(\mathrm{mm}^{2}\right)$ & $20 \times 15$ & $70 \times 44$ \\
Maximum field $(\mathrm{T})$ & 0.110 & 0.05 \\
Inductance $(\mu H)$ & 1.3 & 0.4 \\
Peak current $(\mathrm{kA})$ & 1.8 & 1.9 \\
Drive voltage $(\mathrm{kV})$ & 0.175 & 15 \\
Pulse shape & 100 s full sine & $100 \mathrm{~ns}$ rise, \\
& & $300 \mathrm{~ns}$ flattop, \\
& & $100 \mathrm{~ns}$ fall \\
\hline \hline
\end{tabular}




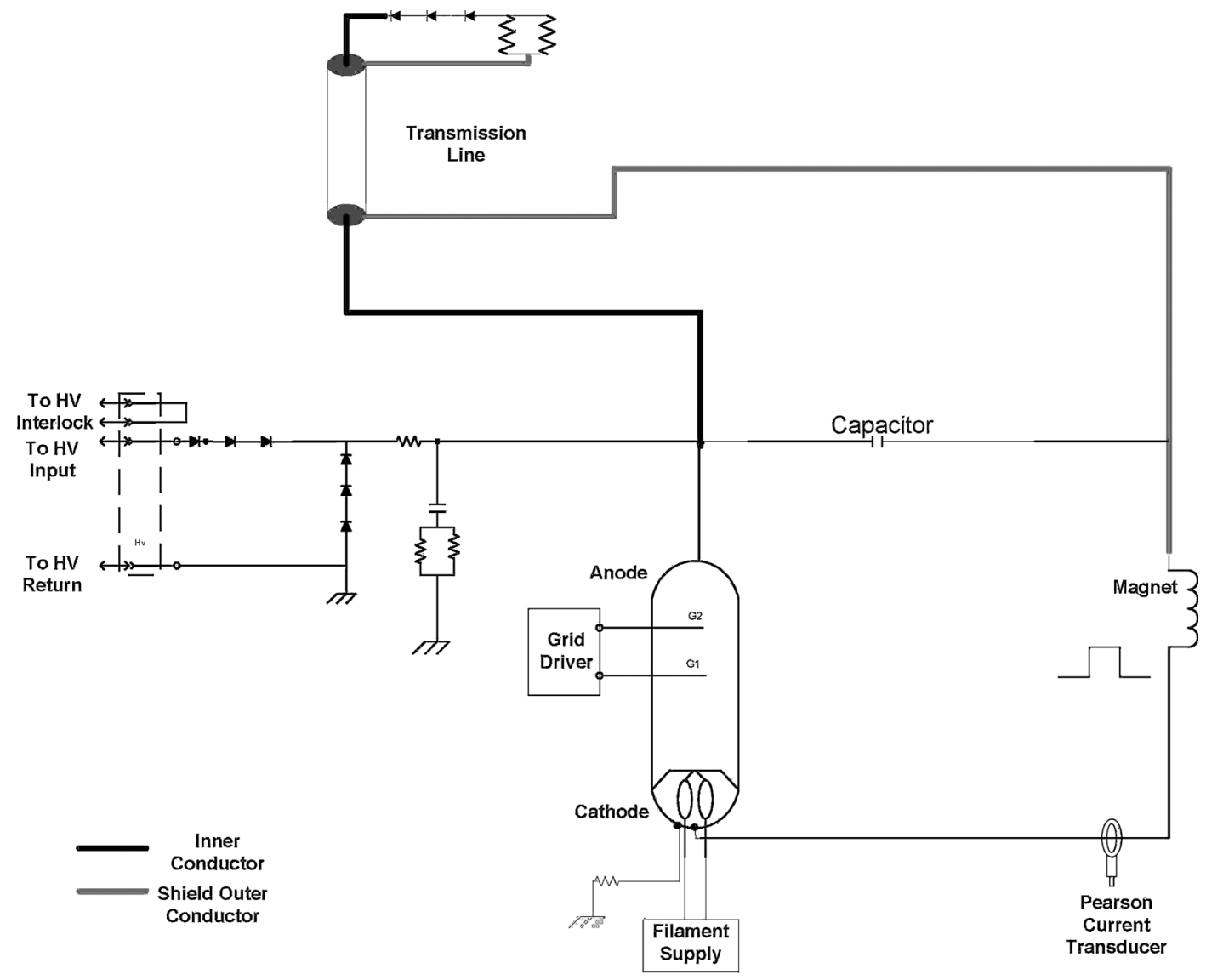

FIG. 9. Booster injection kicker driver block diagram.

\section{BOOSTER RAMP WITH SINGLE TRAIN INJECTION}

The first step in understanding the expected performance of the stacking scheme is to understand the dynamics of the beam on the ramp without stacking. The booster repetition rate is $1 \mathrm{~Hz}$. The simulations in this paper assume that the
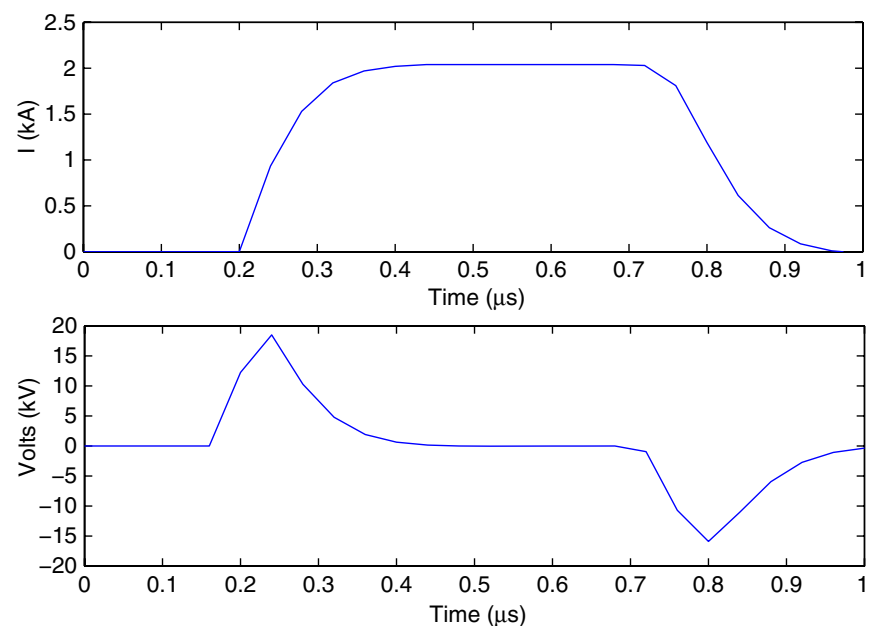

FIG. 10. Booster injection kicker simulated waveform. The upper plot is the current through the kicker, and the lower plot is the voltage across the kicker. booster ramp is a half sine with a period of $800 \mathrm{~ms}$. This leaves $200 \mathrm{~ms}$ for accumulating beam in the booster. The rf voltage and phase are varied accordingly to increase the energy of the beam and replace synchrotron radiation losses while slowly lowering the synchrotron frequency. The rf frequency is locked to the storage ring and is constant through the ramp. Figure 12 shows the energy, voltage, and phase ramps from injection to extraction.

The $400 \mathrm{~ms}$ ramp corresponds to 757000 turns. The simulated beam consisted of 1000 particles with transverse rms emittances of $155 \mathrm{~nm}$. This contains $87 \%$ of the particles in the anticipated beam distribution. The energy spread is $0.5 \%$, and the bunch length is $3 \mathrm{~mm}$. One bare lattice along with 20 seeds to generate lattices with errors

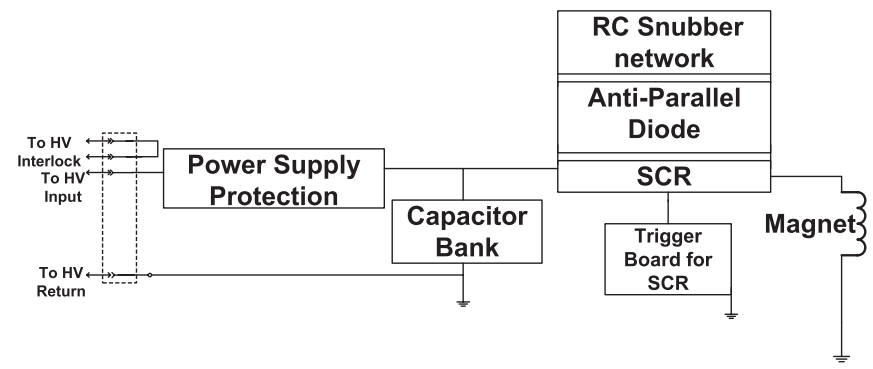

FIG. 11. Booster injection septum driver block diagram. 

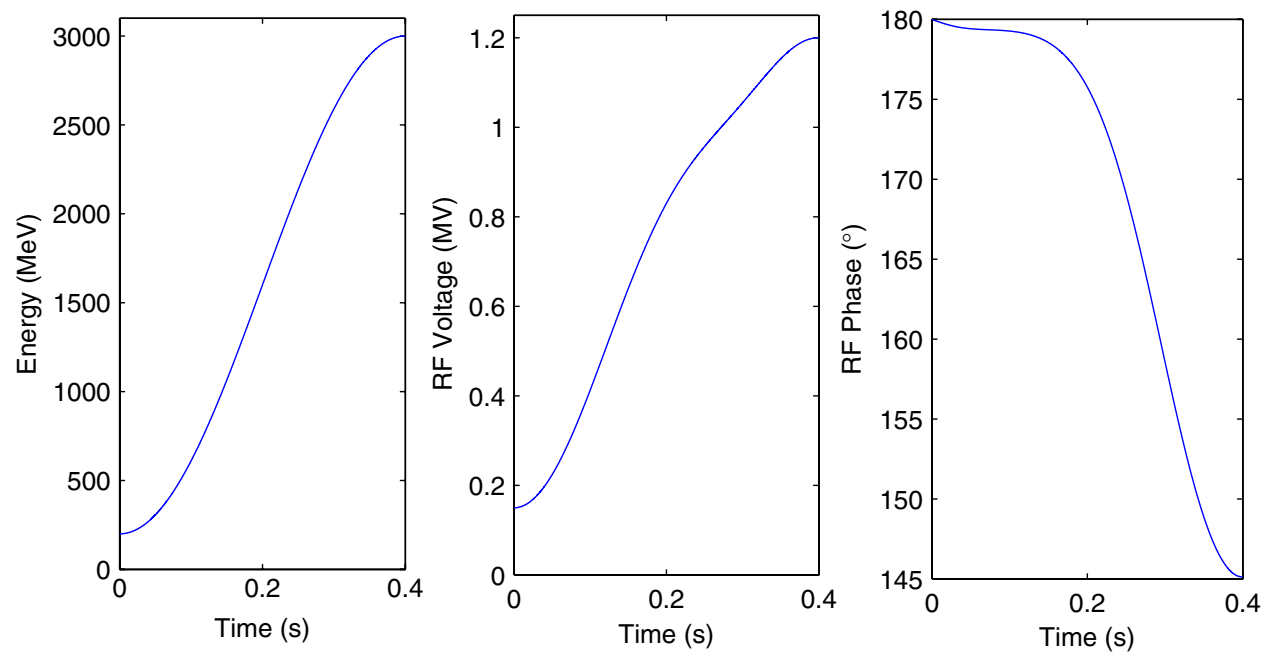

FIG. 12. The energy (left), rf voltage (center), and phase (right) ramp in the booster.

were simulated. The orbit was corrected with an average maximum deviation of $1.5 \mathrm{~mm}$ in the horizontal plane and $1.2 \mathrm{~mm}$ in the vertical plane. No tune or chromaticity correction are performed.

The bare booster lattice has a $99.5 \%$ charge transport efficiency through the ramp. Sixteen of the 20 seed lattices with errors had an transport efficiency greater than $98 \%$. The lowest efficiency was $88.7 \%$ for single train injection. Further analysis of the beam loss shows that most of the losses occur within the first $50 \mathrm{~ms}$ of the ramp, which corresponds to an energy gain of $300 \mathrm{MeV}$. Additional losses from beam scattering on the residual gas are expected to be on the order of $0.5 \%$. Figure 13 shows a typical seed's charge transmission through the ramp and energy as a function of time.

The average horizontal emittance is $39 \pm 2 \mathrm{~nm}$, and energy spread is $0.080 \pm 0.002 \%$ at extraction. The vertical emittance has a range of $0.2 \mathrm{~nm}$ to $3.1 \mathrm{~nm}$. Figure 14

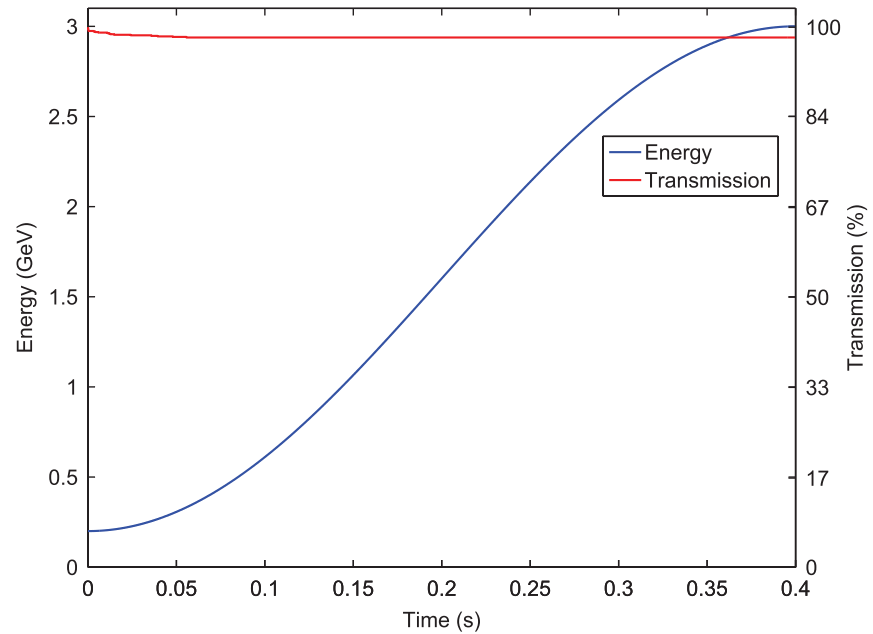

FIG. 13. The energy and transmission vs time for the booster ramp with a single bunch. shows the horizontal emittance through the ramp for one of the seeds and compares with theory. This dependence is in good agreement with numerical solution of the differential equation describing beam damping and quantum excitation based on Sands-Kolomensky-Lebedev theory $[15,16]$.

\section{BEAM STACKING}

Stacking of the beam relies on maintaining sufficient stay clear aperture in the booster to minimize the losses of the two beams. Closed orbit distortions reduce the amount of available aperture. Injecting the first beam on the closed orbit is important to maintain the beam emittance. If there is significant emittance blowup or beam loss between injection of the first bunch train and when the second bunch enters, then stacking scheme could be compromised. Increased emittance of the first beam also increases the separation between the trains which increases the

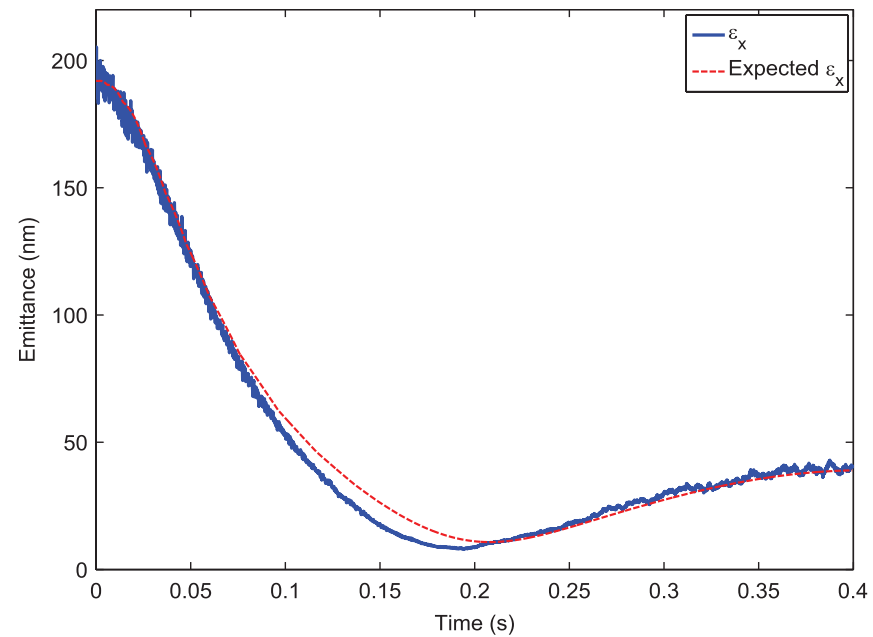

FIG. 14. Simulations of the horizontal emittance on the ramp for a lattice with errors and orbit correction. 
emittance of the combined beam. We considered misalignment, field, and power supply ripple errors in the linac to booster transfer line along with linac energy errors. These result in a two sigma deviation from the closed orbit of $\pm 0.62 \mathrm{~mm}$ and $\pm 0.16 \mathrm{mrad}$ which leads to an emittance increase of 3.4 for the first beam. This amounts to almost doubling the rms beam size, which is still within acceptable limits. We also wanted to quantify the effect of power supply ripple on the closed orbit distortion. For each of the seeds we added $0.1 \%$ relative errors on the booster power supplies. An additional ten seeds for each of the above lattices were generated assuming each quadrupole family is driven from a common supply and each dipole family is driven off of two separate supplies. These 200 seeds resulted in a maximum horizontal orbit deviation of $2 \mathrm{~mm}$, comparable with the $2.6 \mathrm{~mm}$ deviation without the power supply error. The power supply variation was small on top of the errors in Table I and produced a negligible effect so was not used in the further analysis.

The linac repetition rate is $10 \mathrm{~Hz}$, which corresponds to 189000 turns in the booster. We simulated the first beam circulating in the booster for 189000 turns with no injection errors. A bare lattice as well as 20 seeds with errors were used. A bunch with transverse emittance of $155 \mathrm{~nm}$, $0.5 \% \mathrm{rms}$ energy spread, and $3 \mathrm{~mm}$ bunch length containing 1000 particles was simulated in each seed. The transverse phase spaces were matched to the booster. The bare lattice saw no particle loss. The beam loss was less than $2 \%$ for all but six seeds. Lost particles were primarily those located in the beam halo. Beam loss due to beam gas scattering is expected to be $1 \%$. This would increase the expected beam loss between linac pulses to be approximately $3 \%$.

When the second bunch train enters the injection septum we want the centers to be separated by

$$
\Delta x=3 \sigma_{x, 1}+3 \sigma_{x, 2}+5 \mathrm{~mm} .
$$

Subscripts 1 and 2 denote the circulating train and the newly injected train, respectively. The anticipated septum knife width is $3 \mathrm{~mm}$. We desire $1 \mathrm{~mm}$ between each beam edge and the septum knife to leave room for orbit corrections. This is the origin of the $5 \mathrm{~mm}$ additional separation. We want to leave clearance for $3 \sigma_{x}$ for each train to ensure good injection efficiency. Ultimately this separation is determined by the ripple of the first two injection bumps, which places the circulating beam near the septum, and the motion of the injected beam relative to the septum. The $2 \%$ ripple on the first two injection bumps moves the circulating beam $\pm 0.67 \mathrm{~mm}$ and $\pm 0.23 \mathrm{mrad}$ if we consider twice the rms deviations. The injection errors on the second beam $\pm 0.58 \mathrm{~mm}$ and $\pm 0.04 \mathrm{mrad}$ at the septum. The tolerance of $1 \mathrm{~mm}$ clearance at the septum seems reasonable considering the expected errors.

The ripple on the second pair of injection bumps moves the combined bunch centroid from closed orbit $\pm 0.56 \mathrm{~mm}$ and $\pm 0.31 \mathrm{mrad}$ The combination of all of the injection errors is shown in Fig. 15. When all of the injection errors are considered along with the reduced booster acceptance from closed orbit deviations, there is still ample phase space for stacking in the booster.

The dynamics of the stacked trains along the ramp were simulated assuming no injection errors. Two identical bunches were generated and centers were separated as in Eq. (1). Each bunch contained 500 particles, $0.5 \%$ rms energy spread, and $3 \mathrm{~mm}$ bunch length. The transverse phase spaces of each bunch were matched to the booster lattice.

We wanted to study the stacking scheme for a variety of emittances and bunch separations to ensure the robustness of the system. Table III lists the separations and transverse emittances that were used to simulate the effects of different emittances and bunch separations. Scenario 0 is the single beam ramp, included for comparison. The other scenarios are ordered by the combined beam phase space. Scenario 4 is the design parameter set for stacking in the booster. In this scenario the linac emittance is within specification and the bunches are placed as close together as possible. These different scenarios will allow us to study the effects of the linac emittance and bunch separation on beam stacking. A larger linac emittance will require a larger transverse separation between the bunches which requires more of the booster acceptance.

Twenty seeds were used to simulate the booster ramp. Four of them exhibited bad transmission (less than 80\%) in all scenarios listed above and were not used in the analysis. If the booster exhibited the poor charge transmission that occurs in these seeds, the root causes would be rectified prior to attempting beam stacking. Figure 16 shows the horizontal phase space of the two bunches after injection of

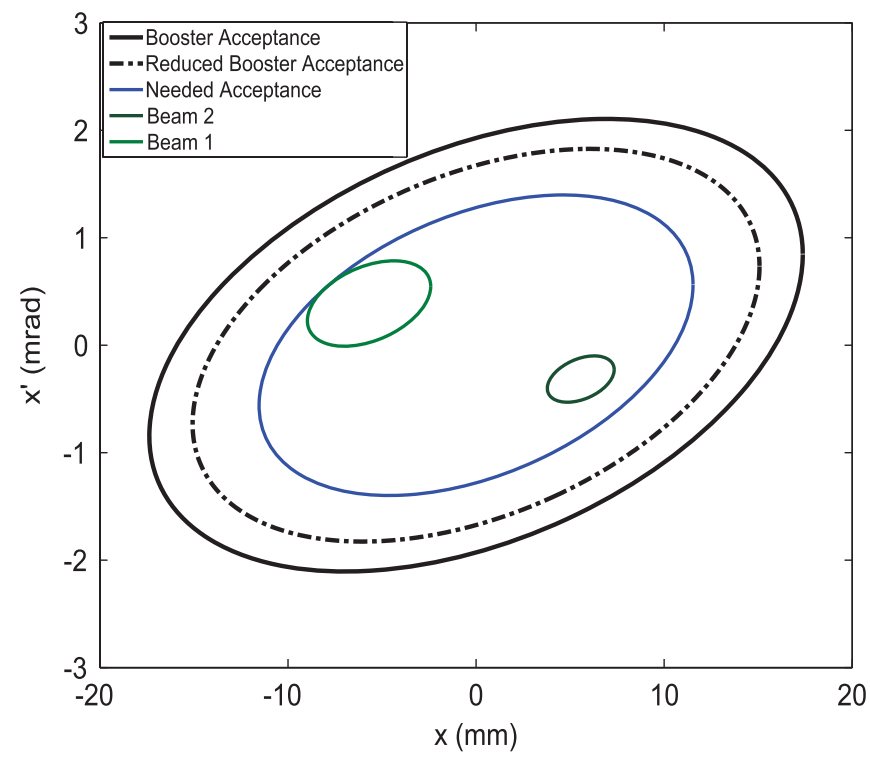

FIG. 15. The acceptance of the booster with injection and closed orbit errors. 
TABLE III. Various injected bunch parameters using in stacking simulations.

\begin{tabular}{lcccc}
\hline \hline Scenario & $\begin{array}{c}\text { Emittance } \\
(\mathrm{nm})\end{array}$ & $\begin{array}{c}\text { Separation } \\
(\mathrm{mm})\end{array}$ & $\begin{array}{c}\text { Occupied phase } \\
\text { space }(\mathrm{mm} \text { mrad })\end{array}$ & Transmission \\
\hline 0 & 155 & 0 & 3.9 & $99 \pm 1 \%$ \\
1 & 155 & 13 & 18.9 & $91 \pm 9 \%$ \\
2 & 39 & 13 & 14.1 & $93 \pm 9 \%$ \\
3 & 78 & 11.4 & 13.8 & $95 \pm 7 \%$ \\
4 & 39 & 10.5 & 10.9 & $96 \pm 6 \%$ \\
5 & 25 & 10.14 & 9.7 & $96 \pm 5 \%$ \\
\hline \hline
\end{tabular}

the second bunch and at extraction for a seed in scenario 4. The lost particles are shown in red on the left plot. The horizontal emittance of the beam at extraction is $39 \mathrm{~nm}$, and the vertical emittance is $1.6 \mathrm{~nm}$. This is consistent with single beam injection.

Figure 17 shows the simulated transmission of the beam through the ramp as function of the horizontal emittance of the combined beam. The single beam case is shown in black and the stacking specification (scenario 4) is shown as a red star. The points are the mean transmission for each condition and the error bars represent the rms value of the transmission. The transmission through the ramp decreases gradually from $99 \%$ to $91 \%$ as the combined beam emittance increases a factor of 1.9. As stated above, the horizontal acceptance of the booster is $31 \mathrm{~mm}$ mrad. All considered cases are 39\% smaller than this limit.

Table IV tabulates the losses encountered by the single bunch train and double bunch train during the ramping and stacking process. Approximately $97 \%$ of the initial beam remains at the injection of the second beam. Approximately $95 \%$ of the stacked trains survive the

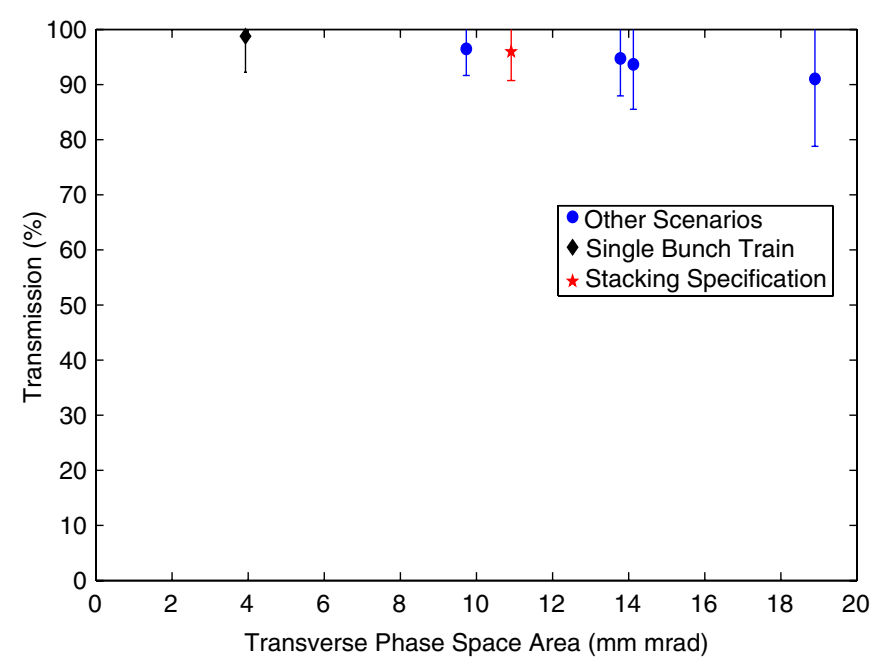

FIG. 17. Transmission through the ramp as a function combined beam horizontal emittance. The black diamond shows the single beam case, the red star shows scenario 4 , the specification for beam stacking.
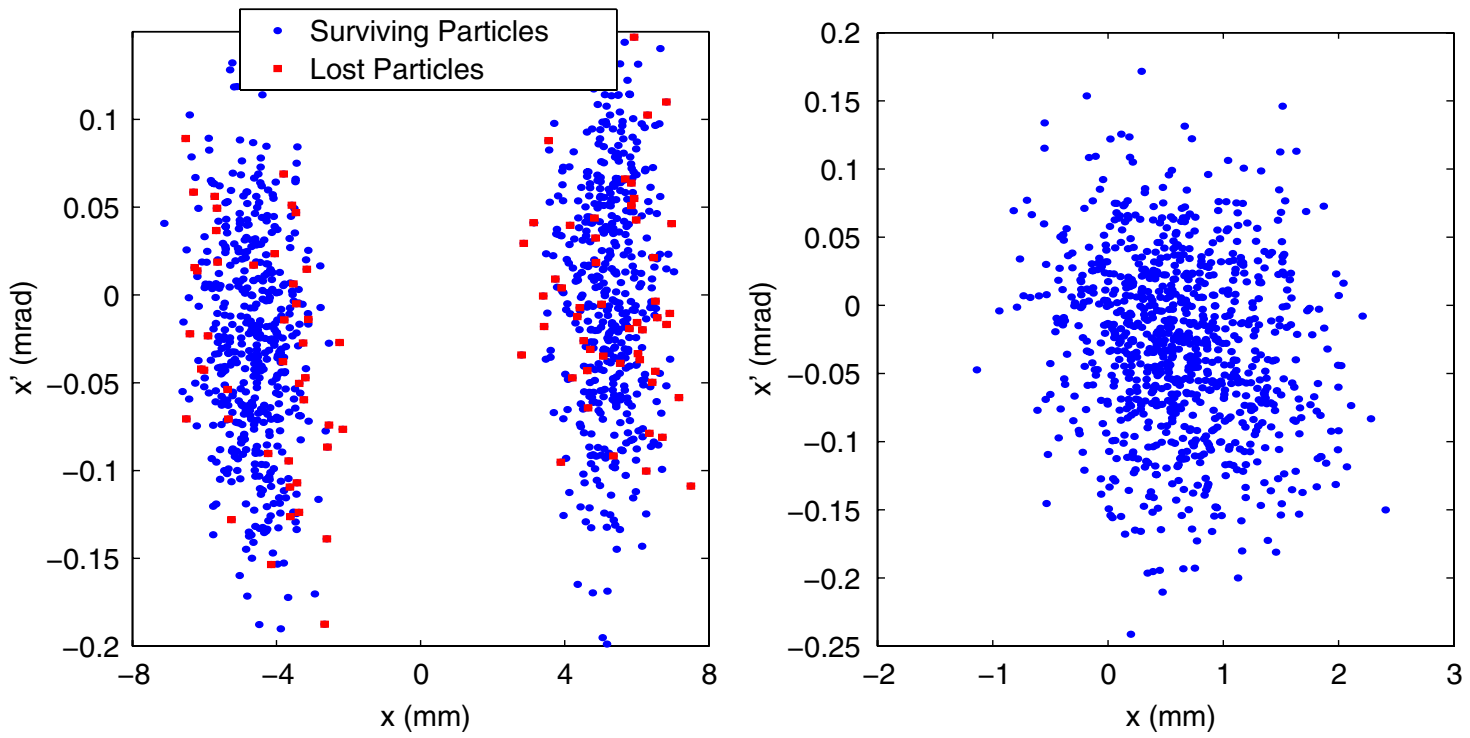

FIG. 16. Injection (left) and extraction (right) horizontal phase spaces at the end of the injection straight for a seed in scenario 4. Lost particles are shown in red on the left plot. 
TABLE IV. Losses during the stacking and ramping process for two beams for the design stacking parameters.

\begin{tabular}{lc}
\hline \hline Loss mechanism & Amount \\
\hline Beam gas scatter for first beam & $1 \%$ \\
First beam losses on aperture & $2 \pm 1 \%$ \\
Two beam loss on aperture during ramp & $4 \pm 6 \%$ \\
Two beam vacuum loss & $1 \%$ \\
Total two beam loss & $5 \pm 6 \%$ \\
\hline \hline
\end{tabular}

ramp for scenario 4 . This equates to $94 \%$ transmission of each bunch. This should be compared to $98 \%$ transmission for a single bunch through the ramp. The result of beam stacking is to reduce the total charge requirement of the linac from 15 to $8 \mathrm{nC}$

\section{CONCLUSION}

In this paper, we detail our studies of beam stacking in the NSLS-II booster, and the corresponding minimal requirements and tolerances for the injector parameters. Our approach is a robust solution that will double the injector's performance without increasing the size or power of the machine.

A four kicker system was designed to stack the bunch trains next to each other while keeping the beams inside the booster acceptance. We developed a model of the booster ramp with $99 \pm 1 \%$ transmission of a single beam including machine errors. We simulated this ramp for the stacked beam and show that at the specified linac emittance and beam separation the two beam transmission is $96_{-6}^{+4} \%$. Once the losses for the first beam and beam gas scattering are considered, the stacking scheme accelerates the equivalent of $94 \%$ of each bunch to $3 \mathrm{GeV}$.

The idea of stacking three bunch trains transversely was investigated. The maximum extent of the three beams would be approximately $35 \mathrm{~mm}$ at the end of the injection straight. This is larger than the booster stay clear aperture of $30 \mathrm{~mm}$. For this reason, stacking three beams in the booster is no longer being investigated.

The stacking scheme will reduce the charge requirement from the linac from 15 to $8 \mathrm{nC}$. The booster design is robust enough that, even if the linac emittance should suffer, the stacking scheme will still be successful. Because of these successful studies, we are implementing this scheme in the NSLS-II booster.

\section{ACKNOWLEDGMENTS}

The authors would like to thank Robert Meier for providing the technical drawings in Figs. 6 and 7. We would also like to thank Riccardo Fabris of Trieste and Pierre Lebasque of SOLEIL for their help with the injection kicker driver design. This manuscript has been authored by Brookhaven Science Associates, LLC under Contract No. DE-AC02$98 \mathrm{CH} 10886$ with the U.S. Department of Energy.

[1] Y. Kamiya, Elementary Analysis of Phase Space Painting (IEEE, Chicago, 1989).

[2] J. D. Galambos, J. A. Holmes, Y. Y. Less, A. Luccio, D. K. Olsen, and J.J. Beebe-Wang, in Proceedings of the 6th European Particle Accelerator Conference, Stockholm, 1998 (IOP, London, 1998), MOP49C.

[3] D. Boussard and Y. Mizumachi, IEEE Trans. Nucl. Sci. 26, 3623 (1979).

[4] C. Ankenbrandt, Fermilab Report No. FN-325, 1981.

[5] S. Hansen, K. N. Henrichsen, and M. de Jonge, IEEE Trans. Nucl. Sci. 20, 805 (1973).

[6] NSLS-II Preliminary Design Report, 2007, http:// www.bnl.gov/nsls2/project/PDR/.

[7] T. Shaftan, J. Rose, R. Heese, N. Tsoupas, Y. Li, W. Guo, D. Hseuh, E. D. Johnson, R. Meier, I. Pinayev, A. Blednykh, S. Krinsky, J. Skaritka, F. J. Willeke, G. Ganetis, S. Ozaki, S. Sharma, M. Rehak, and O. Singh, in Proceedings of the 11th European Particle Accelerator Conference, Genoa, 2008 (EPS-AG, Genoa, Italy, 2008), WEPC089.

[8] R. P. Fliller, III, R. Alforque, R. Heese, R. Meier, J. Rose, T. Shaftan, O. Singh, and N. Tsoupas, in Proceedings of the 23rd Particle Accelerator Conference, Vancouver, Canada, 2009 (IEEE, Piscataway, NJ, 2009), TU5RFP006.

[9] T. Shaftan, R. Fliller, R. Heese, J. Skaritka, J. Rose, S. Sharma, G. Ganetis, B. Dalesio, and D. Hseuh, NSLS-II Technical Report No. 61.

[10] R. P. Fliller, III, W. Guo, R. Heese, Y. Li, and T. Shaftan, in Proceedings of the 23rd Particle Accelerator Conference, Vancouver, Canada, 2009 (Ref. [8]), TU5RFP007.

[11] A. Setty, D. Jousse, J.-L. Pastre, F. Rodriguez, A. Sacharidis, R. Chaput, J.-P. Pollina, B. Pottin, and M.-A. Tordeux, in Proceedings of the 10th European Particle Accelerator Conference, Edinburgh, Scotland, 2006 (EPSAG, Edinburgh, Scotland, 2006), TUPCH112.

[12] Request for Proposal No. 153810 National Synchrotron Light Source II (NSLS-II) Electron Linear Accelerator (LINAC).

[13] R. P. Fliller, III, NSLS-II Technical Note 54.

[14] R. Heese, R. P. Fliller, III, R. Meier, B. Parker, M. Rehak, T. Shaftan, F. J. Willeke, P. Zuhoski, and E. Weihreter, in Proceedings of the 23rd Particle Accelerator Conference, Vancouver, Canada, 2009 (Ref. [8]), TU5RFP009.

[15] M. Sands, SLAC-121, 1970.

[16] A. A. Kolomensky and A. N. Lebedev, Theory of Cyclic Accelerators (North-Holland, Amsterdam, 1966). 